Proceedings of the 12th International Symposium on Physics of Materials, Prague, September 4-8, 2011

\title{
[100] Loading of Fcc Based Nanofibre Reinforced Composites
}

\author{
M. ČERNÝ* AND J. POKLUdA \\ Faculty of Mechanical Engineering, Brno University of Technology \\ Technická 2, Brno 616 69, Czech Republic
}

\begin{abstract}
$A b$ initio calculations of theoretical tensile and compressive strengths in the $\langle 100\rangle$ direction of a composite having continuous nanofibre reinforcements are performed using pseudopotential approach within density functional theory. Results for iridium or platinum nanofibres in copper or gold matrices, presented as case studies, reveal that the theoretical tensile and compressive strengths almost linearly increase with increasing atomic concentration of the reinforcement exhibiting only slight positive strength deviations from the linearity for $\mathrm{Pt}-\mathrm{Au}$ and slight negative deviations of the tensile strength for $\mathrm{Ir}-\mathrm{Cu}$.
\end{abstract}

PACS: $61.46 . \mathrm{Km}, 62.20 . \mathrm{D}-, 62.23 . \mathrm{Pq}, 68.35 . \mathrm{bd}, 81.05 . \mathrm{Ni}$

\section{Introduction}

Some of the physical or mechanical properties of fibre-reinforced composites are usually calculated using simple empirical linear mixture rules that predict resulting values of particular quantities (e.g. the Young modulus) from values measured for crystals of the constituent elements and from the volume fraction of fibres. Theoretical atomistic studies [1] on bcc based nanocomposite models exhibiting a perfect cohesion on the fibre/matrix interface revealed that the linear mixture rules are valid at the atomistic level e.g. for values of the equilibrium volume and the bulk moduli. In case of the theoretical tensile strength (TTS), however, the obtained data predicted remarkable strength enhancement (particularly at $60-90 \%$ concentration of reinforcement fibres) in the composite. This means that the strength values computed for certain composite was greater than the estimate based on the linear mixture rule. In some cases, the predicted TTS of the nanocomposite was even slightly higher than that of the reinforcement itself.

Since one of possible explanations dwelled in an effect of transverse stresses (caused by incompatibility strains) acting on the matrix and fibres, this effect was systematically studied not only for the $\langle 100\rangle$ crystallographic direction [2] (relevant to the model of nanocomposite loading) but also for tension in $\langle 110\rangle$ and $\langle 111\rangle$ directions [3]. In most cases, the transverse tensile stresses raised the maximum axial stress (i.e. TTS). Sensitivity of TTS to the transverse stresses can be roughly estimated from TTS under purely uniaxial load and from the theoretical isotropic strength as was demonstrated in Ref. [3]. In the case of uniaxial compression, the effect of transverse stresses was found to be opposite and even stronger [4]. Thus, the aforementioned stress-synergy effect could play the major part in explanation of the strength enhancement in models of nanocomposite structures.

\footnotetext{
* corresponding author; e-mail: cerny.m@fme.vutbr.cz
}

In this paper, we verify existence of the synergy effect also in two models of fcc based nanocomposites. The theoretical strengths in tension as well as in compression are computed for different thickness of nanofibres (from a single atom to several atomic distances) by means of $a b$ initio method for Ir fibres in $\mathrm{Cu}$ matrix and $\mathrm{Pt}$ fibres in Au matrix.

\section{Computational procedure}

A model of the nanocomposite is built as a periodic repeating of $2 \times 2 \times 1$ fcc-based supercell displayed in Fig. 1 . The crystal basis of our supercell contains 16 atoms in total (ordered in two neighbouring $\{001\}$ planes). The coordinate system is oriented to make the translational vectors $\boldsymbol{p}_{1}, \boldsymbol{p}_{2}$, and $\boldsymbol{p}_{3}$ parallel to [100], [010], and [001] crystallographic directions of the fcc cells, respectively. Occupying the lattice sites by atoms of either fibre or matrix material enables us to model infinite fibres of various thickness (oriented along the [001] direction) equidistantly spaced in the composite model. Of course, the atomic concentration of fibre material increases with increasing fibre thickness. The computational supercell in Fig. 1 represents a basic unit for building the nanocomposite model with $5 / 16=31.3 \%$ atomic concentration of reinforcement. This study considers Ir and Pt as the reinforcing material (since they exhibit higher Young's moduli and TTS) in $\mathrm{Cu}$ or $\mathrm{Au}$ matrices. In order to avoid any shear stresses, the fibres are considered symmetrical which, of course, limits the number of different atomic concentrations of the reinforcement in such a relatively small supercell. Therefore, we studied only three different concentrations, namely, $5 / 16,9 / 16$, and $11 / 16$ and compared the obtained data with those for a pure matrix $(0 \%)$ and the reinforcement (100\%).

Calculation procedure considered three different approaches: uniaxial deformation, uniaxial loading and restricted loading. The uniaxial deformation means that lateral strains are zero, i.e., the translational vectors $\boldsymbol{p}_{1}$ and $\boldsymbol{p}_{2}$ are kept constant for all values of axial strain (all 


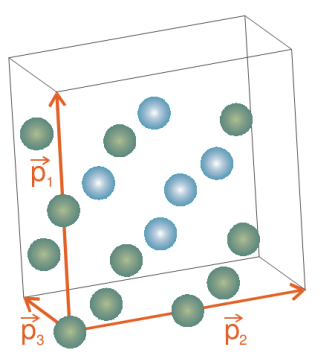

Fig. 1. A model of the supercell with displayed primitive translational vectors $\boldsymbol{p}_{1}, \boldsymbol{p}_{2}$ and $\boldsymbol{p}_{3}$.

lengths of $\boldsymbol{p}_{3}$ ). During the uniaxial loading, the lateral stresses were relaxed by optimizing lengths of the translational vectors $\boldsymbol{p}_{1}$ and $\boldsymbol{p}_{2}$. This also includes full optimization of the structure (relaxing also the forces computed for individual atoms). The restricted loading represents a uniaxial loading with a fixed basis (with equidistant arrangement of atoms in planes perpendicular to the loading axis).

Using this procedure, the uniaxial loading or deformation was applied parallel to the lamina fibres. The dependence of the uniaxial stress $\sigma$ on the engineering strain $\varepsilon$ was used for calculation of the Young modulus $E_{001}$ according to the Hooke law and for identification of the stress maxima in tension and compression. If no other instabilities precede, the stress maxima can be considered to be the theoretical tensile and compressive strengths of the nanocomposite.

Under $\langle 100\rangle$ compression, the fcc lattice transforms via tetragonal Bain's path to bcc. Since this bcc state is a stress-free state that corresponds to symmetry dictated energy maximum the compressive stress reaches its maximum prior approaching this state and the compressive strength is limited by the structural transformation. On the other hand, there is no state of higher lattice symmetry during uniaxial tension.

The forces and stresses for the relaxation procedure were calculated according to the Hellman-Feynman theorem using the plane wave code Vienna Ab initio Simulation Package (VASP) [5]. We utilized the ultra-soft pseudopotentials of the Vanderbild type implemented within the VASP code. Cut-off energy for the basis set was $310 \mathrm{eV}$ and $260 \mathrm{eV}$ for $\mathrm{Ir}-\mathrm{Cu}$ and $\mathrm{Pt}-\mathrm{Au}$ systems, respectively. The exchange-correlation energy was evaluated using both the local density approximation $(\mathrm{Pt}-\mathrm{Au})$ and the generalized-gradient approximation of Perdew and Wang [6] $(\mathrm{Ir}-\mathrm{Cu})$ and the $6 \times 6 \times 12 k$-points mesh was used in all our calculations. The solution was considered to be self-consistent when the energy difference of two consecutive iterations was smaller than $0.1 \mathrm{meV}$.

\section{Results}

In order to verify reliability of the applied computational procedure, the equilibrium lattice parameter $a_{0}$, the Young modulus $E_{001}$ and the Poisson ratio $\nu_{001}$ were calculated for single crystals of $\mathrm{Cu}$, Ir, Pt and $\mathrm{Au}$ using the above described supercells with all atomic positions occupied by atoms of the same kind (in this case, the uniaxial loading and restricted loading are equivalent). The obtained values are listed in Table I along with experimental data. The experimental values of $a_{0}$ were taken from Ref. [7] and $E_{001}$ as well as $\nu_{001}$ values were computed from elastic moduli $[7,8]$. The agreement of computed and experimental $a_{0}$ values is within $1 \%$. The agreement in the case of elastic moduli and Poisson's ratios is mostly also good (within 10\%), however, computed Young's moduli for $\mathrm{Cu}$ and $\mathrm{Au}$ remarkably overestimate the experimental data.

TABLE I

Computed and experimental values of the equilibrium lattice parameter $a_{0}$, the Young modulus $E_{001}$ and the Poisson ratio $\nu_{100}$ for pure $\mathrm{Cu}, \mathrm{Ir}, \mathrm{Pt}$, and $\mathrm{Au}$.

\begin{tabular}{c|c|c|c|c|c|c}
\hline \hline \multirow{2}{*}{ Element } & \multicolumn{2}{|c|}{$a_{0}[\mathrm{~nm}]$} & \multicolumn{2}{c|}{$E_{001}[\mathrm{GPa}]$} & \multicolumn{2}{c}{$\nu_{001}$} \\
\cline { 2 - 7 } & calc. & exp. & calc. & exp. & calc. & exp. \\
\hline $\mathrm{Cu}$ & 3.64 & $3.61^{a}$ & 87 & $73^{b}$ & 0.40 & $0.42^{b}$ \\
$\mathrm{Ir}$ & 3.88 & $3.83^{a}$ & 443 & $438^{a}$ & 0.28 & $0.29^{a}$ \\
$\mathrm{Pt}$ & 3.91 & $3.91^{a}$ & 144 & $136^{a}$ & 0.42 & $0.42^{a}$ \\
$\mathrm{Au}$ & 4.07 & $4.07^{a}$ & 59 & $46^{b}$ & 0.44 & $0.46^{b}$ \\
\hline
\end{tabular}

${ }^{a}$ Ref. [7], ${ }^{b}$ Ref. [8]

\section{TABLE II}

Computed maximum tensile $\sigma_{\max }^{+}$and compressive $\sigma_{\max }^{-}$stresses and the corresponding strains $\varepsilon_{\max }^{+}$and $\varepsilon_{\max }^{-}$along with data from literature.

\begin{tabular}{c|c|c|c|c|c|c|c|c}
\hline \hline \multirow{2}{*}{ Element } & \multicolumn{2}{|c|}{$\sigma_{\max }^{+}[\mathrm{GPa}]$} & \multicolumn{2}{|c|}{$\varepsilon_{\max }^{+}$} & \multicolumn{2}{c|}{$\sigma_{\max }^{-}[\mathrm{GPa}]$} & \multicolumn{2}{c}{$\varepsilon_{\max }^{-}$} \\
\cline { 2 - 9 } & calc. $^{-}$liter. $^{a}$ & calc. & liter. $^{a}$ & calc. & liter. & calc. $^{b}$ & liter. $^{b}$ \\
\hline $\mathrm{Cu}$ & 24.2 & 24.1 & 0.36 & 0.36 & -3.7 & -3.6 & -0.09 & -0.10 \\
$\mathrm{Ir}$ & 44.4 & 44.5 & 0.25 & 0.25 & -49.5 & -49.2 & -0.13 & -0.13 \\
$\mathrm{Pt}$ & 34.3 & 34.1 & 0.35 & 0.34 & -7.3 & -7.4 & -1.12 & -0.12 \\
$\mathrm{Au}$ & 18.6 & 18.6 & 0.33 & 0.33 & -1.6 & -1.6 & -0.09 & -0.09 \\
\hline
\end{tabular}

${ }^{a}$ Ref. [2], ${ }^{b}$ Ref. [4] 


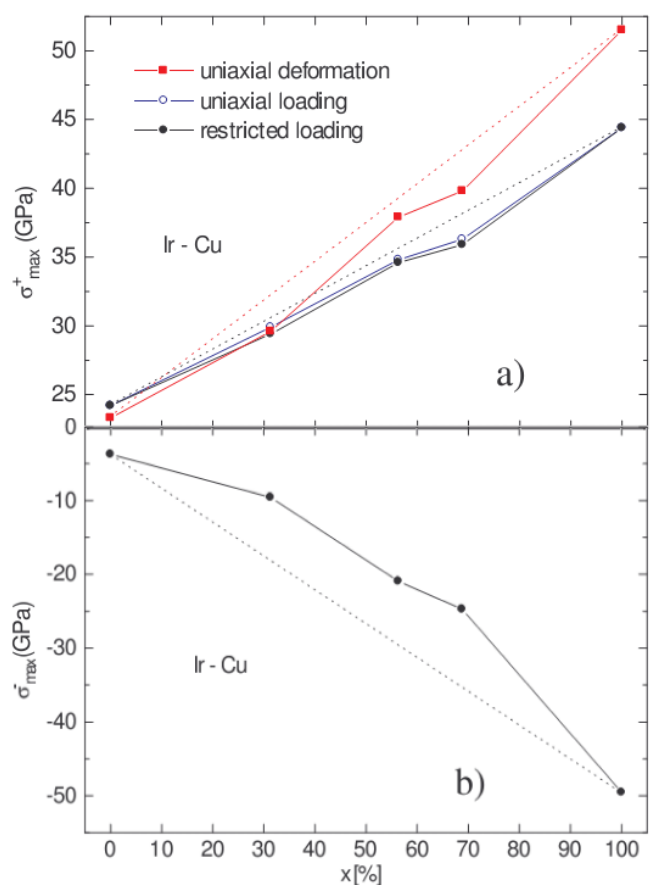

Fig. 2. Dependence of the maximum tensile stress (a) in tension $\sigma_{\max }^{+}$and (b) in compression $\sigma_{\max }^{-}$on atomic concentration $x$ of iridium in $\mathrm{Ir}-\mathrm{Cu}$ nanocomposite.

The maximum tensile $\sigma_{\max }^{+}$and compressive $\sigma_{\max }^{-}$ stresses and the corresponding critical strains $\varepsilon_{\max }^{+}$and $\varepsilon_{\max }^{-}$for pure $\mathrm{Cu}, \mathrm{Ir}, \mathrm{Pt}$ and $\mathrm{Au}$ are collected in Table II together with available literature data $[2,4]$ reported for an equivalent uniaxial loading (using primitive cells). One can note a very good agreement for all listed values.

The maximum tensile $\sigma_{\max }^{+}$and compressive $\sigma_{\max }^{-}$ stresses computed for $\mathrm{Ir}-\mathrm{Cu}$ nanocomposite models of different atomic concentrations of the reinforcement are displayed in Fig. 2. The solid lines are just guide to the eye and the dotted lines represent a linear interpolation between the values computed for bulk crystals of both constituents. The solid boxes in Fig. 2a represent $\sigma_{\max }^{+}$ values calculated for the uniaxial deformation, the open circles represent the model of the uniaxial loading and the solid circles stand for the results of the restricted loading. Of course, the uniaxial loading is an idealized model of real tensile tests. However, as can be clearly seen, the $\sigma_{\max }^{+}$values for uniaxial loading are almost equal to those for the restricted loading. For this reason, all other diagrams contain only results of calculations for the restricted loading that are computationally less demanding. The values computed for uniaxial deformation are mostly higher. It is because the zero lateral strains induce transverse stresses during increase of the axial tensile strain. Since these stresses raise $\sigma_{\max }^{+}$in Ir and slightly lower $\sigma_{\max }^{+}$in $\mathrm{Cu}[2]$ the related solid line in Fig. 2a intersects the other two connecting lines. Notwithstanding, all three approaches consistently predict the $\sigma_{\max }^{+}$values of the nanocomposites to be somewhat lower than pre-

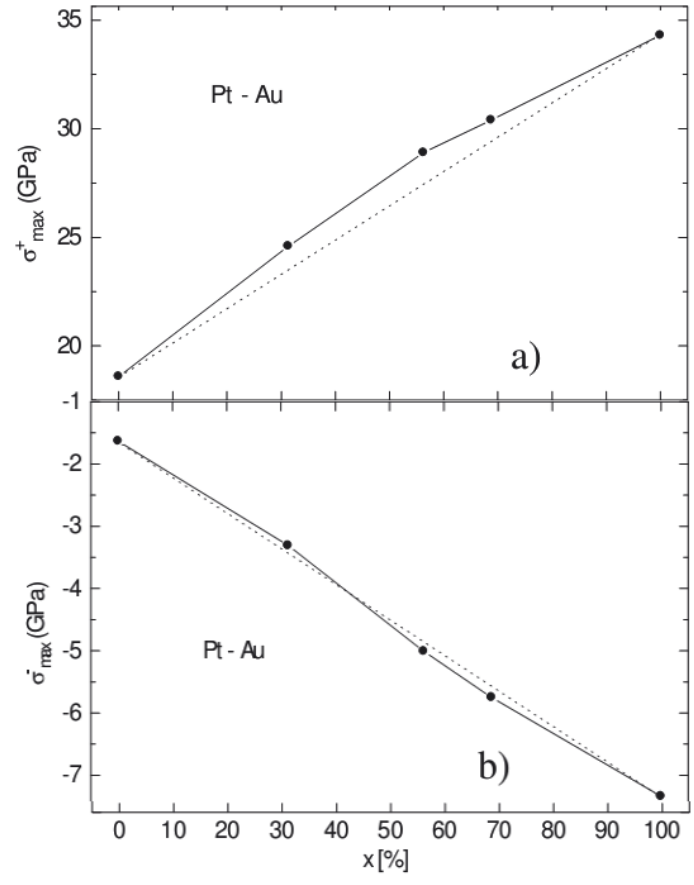

Fig. 3. Dependence of the maximum tensile stress (a) in tension $\sigma_{\max }^{+}$and (b) in compression $\sigma_{\max }^{-}$on atomic concentration of platinum in $\mathrm{Pt}-\mathrm{Au}$ nanocomposite.

dictions based on linear rules of mixture. Thus, in this case, the synergy effect in the composite is negative. The $\sigma_{\max }^{-}$values in Fig. $2 \mathrm{~b}$ also exhibit a negative synergy effect (note that compressive stresses are negative).

Results for the $\mathrm{Pt}-\mathrm{Au}$ system are displayed in Fig. 3. Data for $\sigma_{\text {max }}^{+}$show a positive synergy effect - the data points for composite are slightly but systematically above the interpolating line. On the other hand, the $\sigma_{\max }^{-}$values are more or less aligned along the dotted line which means that no synergy effect was observed in uniaxial compression of this composite.

\section{Summary}

Elasticity and strength of two fcc based nanofibre reinforced composites under uniaxial loading parallel to the fibres were studied from first principles. Computed results for $\mathrm{Ir}-\mathrm{Cu}$ and $\mathrm{Pt}-\mathrm{Au}$ systems reveal that the theoretical strengths almost linearly increase with increasing atomic concentration of the reinforcement exhibiting only a slight strength enhancement (positive synergy) for $\mathrm{Pt}-\mathrm{Au}$ under tensile loading and a slight strength reduction (negative synergy) for $\mathrm{Ir}-\mathrm{Cu}$ under both tension and compression.

\section{Acknowledgments}

Authors acknowledge the financial support of the Czech Science Foundation received within the project GA $106 / 09 / 1524$. 


\section{References}

[1] M. Černý, J. Pokluda, Key Eng. Mater. 465, 73 (2011).

[2] M. Černý, J. Pokluda, Phys. Rev. B 76, 024115 (2007).

[3] M. Černý, J. Pokluda, Phys. Rev. B 82, 174106 (2010).

[4] M. Černý, J. Pokluda, Comput. Mater. Sci. 50, 2257 (2011).

[5] G. Kresse, J. Hafner, J. Phys., Condens. Matter. 6, 8245 (1994); G. Kresse, J. Furthmüller, Phys. Rev. B 54, 11169 (1996).
[6] J.P. Perdew, Y. Wang, Phys. Rev. B 41, 13244 (1992).

[7] Handbook of Chemistry and Physics, Ed. D.R. Lide, CRC Press, Boca Raton 2005.

[8] G. Simmons, H. Wang, Single Crystal Elastic Constants and Calculated Aggregate Properties: A Handbook, MIT Press, Cambridge 1971. 